\title{
Axillary Padding without Drainage after Axillary Lymphadenectomy - a Prospective Study of 299 Patients with Early Breast Cancer
}

\author{
Jean-Rémi Garbay Anne Thoury Etienne Moinon Andréa Cavalcanti Mario Di Palma \\ Guillaume Karsenti Nicolas Leymarie Benjamin Sarfati Françoise Rimareix Chafika Mazouni
}

Department of Breast Surgical Oncology, Institut Gustave Roussy, Villejuif, France

\section{Keywords}

Breast cancer surgery · Axillary padding · Seroma ·

Drainage $\cdot$ Early discharge

\section{Summary}

Background: After lymphadenectomy for early breast cancer, seroma formation is a constant event requiring a suction drainage. This drainage is the strongest obstacle to reducing the hospital stay. Axillary padding without drainage appears to be a valuable option amid the various solutions for reducing the hospital stay. Methods: We conducted a comparison between 114 patients with padding and 185 patients with drainage. Data were obtained from 2 successive prospective studies. Results: The mean hospital stay was 2.4 days (range 1-4) in the padding group and 4.2 days (range $2-9$ ) in the drainage group $(p<0.05)$. There were fewer needle aspirations for seroma in the padding group (8.8 vs. $23 \%, p<0.05)$. At 6 weeks, only $28 \%$ (32/114) of the patients in the padding group reported pain versus $51 \%(94 / 185)$ in the drainage group. The mean pain intensity at 6 weeks was 3 and 4.3 respectively $(p<0.0001)$. Conclusion: Axillary padding without drainage was associated with a better post-operative course than suction drainage in this historical comparison, and the hospital stay was significantly shortened. There are only few series published on this new technique but they all indicate good feasibility and good tolerance. A large randomised multicentric evaluation is now warranted.

\section{Introduction}

Axillary lymphadenectomy (ALN) continues to be the standard option in many cases of breast cancers. In addition to pain, haematoma and infection, the main early adverse effect of ALN is the post-operative production of fluid in the

\author{
Schlüsselwörter \\ Brustkrebsoperation - Axillary Padding · Serom · \\ Drainage $\cdot$ Frühe Entlassung
}

\section{Zusammenfassung}

Hintergrund: Nach Lymphknotendissektion bei primärem Mammakarzinom ist die Serombildung ein konstanter Prozess, der eine Saugdrainage notwendig macht. Diese Drainage ist das Haupthindernis für eine Reduzierung des Krankenhausaufenthaltes. "Axillary Padding" ohne Drainage scheint eine wertvolle Option unter den verschiedenen Möglichkeiten, den Krankenhausaufenthalt zu verkürzen. Methoden: Wir verglichen 114 Patientinnen mit Axillary Padding mit 185 Patientinnen mit Drainage. Die Daten wurden in zwei aufeinanderfolgenden prospektiven Studien gewonnen. Ergebnisse: In der Padding-Gruppe betrug der durchschnittliche Klinikaufenthalt 2,4 (Spanne: 1-4) Tage, in der DrainageGruppe waren es 4,2 (Spanne: 2-9) Tage $(p<0,05)$. In der Padding-Gruppe gab es weniger Serompunktionen (8,8 vs. $23 \%, p<0,05)$. Nach 6 Wochen gaben nur 28\% (32/114) der Patienten in der Padding-Gruppe an, Schmerzen zu haben, gegenüber 51\% (94/185) in der Drainage-Gruppe. Die durchschnittliche Schmerzintensität nach 6 Wochen war 3 und 4,3 ( $p<0,0001$ ). Schlussfolgerung: Axillary Padding ohne Drainage war in diesem Vergleich mit einem besseren postoperativen Verlauf assoziiert als Drainage und der Klinikaufenthalt war signifikant verkürzt. Es gibt nur wenige Publikationen zu dieser neuen Technik, aber diese weisen alle auf eine gute Durchführbarkeit und Tolerierbarkeit hin. Eine große multizentrische Studie sollte nun durchgeführt werden.

axillary basin. Seroma formation is a constant feature but there is a wide variation in the volume. Thus the rate of symptomatic seromas that require needle aspiration ranges from 20 to $60 \%$ [1]. Indeed, simple closure without axillary drainage resulted in a high rate of seroma formation [2] and multiple needle aspirations. Consequently, this option is rarely

\section{KARGER}

Fax +497614520714

Information@Karger.de

www.karger.com
(C) 2012 S. Karger GmbH, Freiburg

1661-3791/12/0073-0231\$38.00/0

Accessible online at:

www.karger.com/brc
Jean-Rémi Garbay, MD

Department of Breast Surgical Oncology

Institut Gustave Roussy

114 rue Edouard Vaillant, 94805 Villejuif Cedex France

Tel. 33 14211-4350, Fax -6036

garbay@igr.fr 
applied and suction drainage has become the rule. It has to be maintained 5-7 days on average [1, 3-5]. It is removed when the daily output is about $50 \mathrm{ml}$ and this is usually a determinant of discharge from hospital. However, pain is induced by the drain itself and needle aspiration cannot be totally avoided. Above all, it has become the main obstacle to a short hospital stay. Modern post-operative care has evolved and is clearly in favour of short hospital stays and out-patient surgery is gaining momentum. This option appears to be well accepted by the patients [6-8]. With about 42,000 new cases per year in France, shorter hospital stays and ambulatory surgery exert a major medico-economic impact [9]. The main alternative option in order to achieve a short hospital stay is early discharge with the drain being maintained at home. But this is not applicable to all patients and is likely to generate further post-operative costs for care and transport. The other option is early removal on the 2 nd or 3rd post-operative day, whatever the daily fluid volume, or even not to insert a drain at all [9]. However, the absence of drainage leads to a high rate of symptomatic seromas that require additional consultations and needle aspirations. Clearly, the best option would be a specific means to substantially reduce seroma formation and to allow us to dispense with the drain. The sole means published to date are biological glue and surgical padding. The purpose of this study was to evaluate a new aponeurosis or fascial padding technique without drainage [10], compared to standard drainage.

\section{Patients and Methods}

\section{Patients}

A total of 299 women who prospectively underwent breast conserving surgery for stage I-II breast cancer, in the Breast Surgical Oncology Department, Institut Gustave Roussy, France, between 2003 and 2005 were studied. The main inclusion criteria were early breast cancer unamenable to sentinel node biopsy but suitable for conservative treatment, with a lymphadenectomy via a separate incision. There was no specific exclusion criterion. This work is a comparison of 2 groups of patients treated successively using 2 distinct axillary drainage techniques during the 2-year study period. The first group of 185 women treated using conventional vacuum drainage was part of a multicentric prospective evaluation of pain after axillary surgery. Unfortunately, this study was never published. We used this group of patients as the control group for this study. With the same methodology, especially the same pain treatment protocol and the same case report form (CRF), we then prospectively studied a group of 114 women treated with axillary padding without drainage.

\section{Surgical Procedures}

The lumpectomy was performed through a separate incision, without drainage. In the control group, level I and II axillary lymph nodes were removed and a suction drain was inserted. The drain was removed when daily fluid flow was less than $50 \mathrm{ml}$ per $24 \mathrm{~h}$. In the padding group, the skin incision was always horizontal. The clavipectoral fascia was incised horizontally. The same axillary clearance was performed, beneath the fascia. Then padding of the axilla was performed, as previously described [10] with three 2.0 polyglactin stitches suturing the two fascia margins to the underlying pectoralis major, serratus anterior and latissimus dorsi

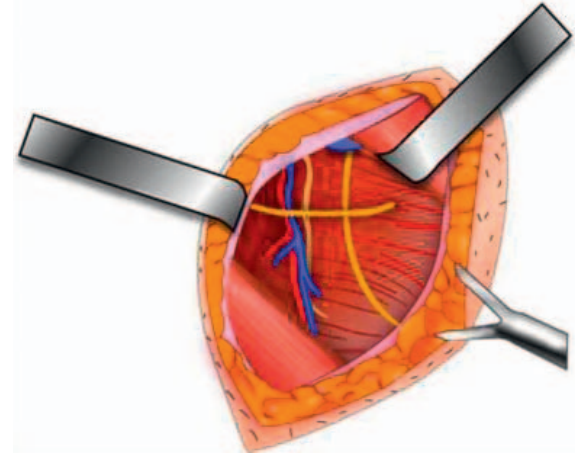

Fig. 1. View after lymphadenectomy, before padding.
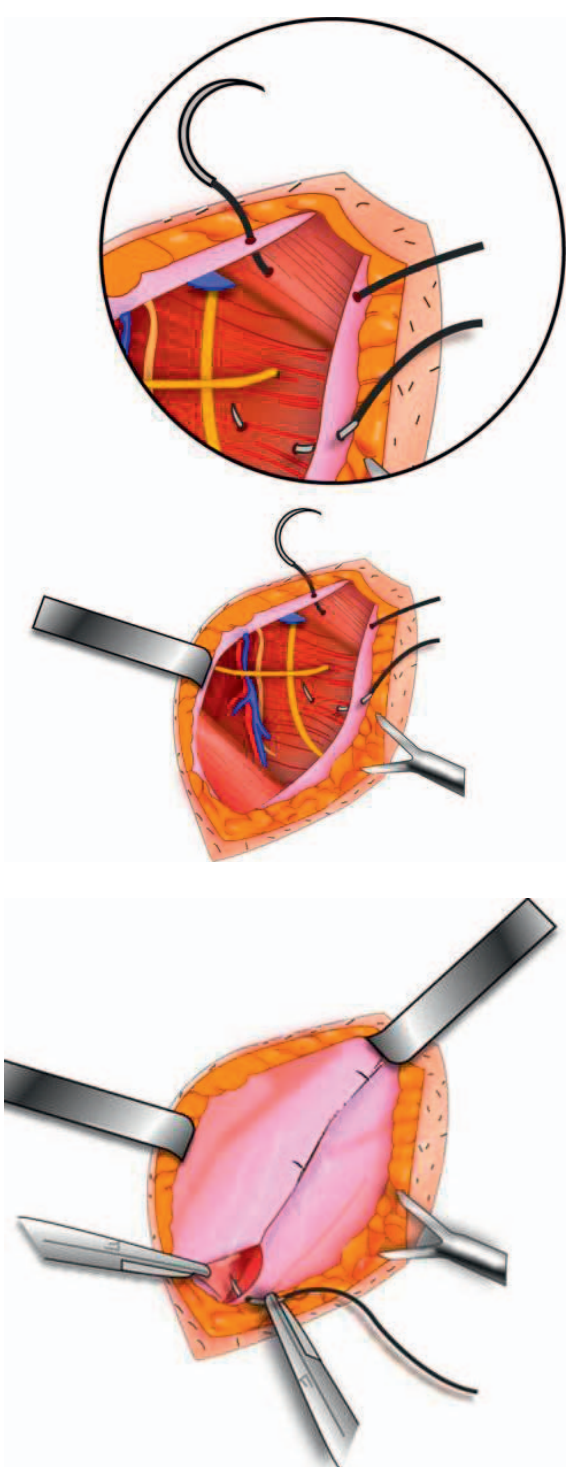

Fig. 2. Aponeurosis padding: first and second stitches.

Fig. 3. Aponeurosis padding: last stitch.

muscles (fig. 1-3). Then, 2-layer skin closure was performed without any skin padding. No compressive dressing was applied in either group. Active mobilisation of the shoulder was allowed on the first post-operative day, and physiotherapy was systematically prescribed after the first week. The same analgesia protocol was used for both groups: sufentanil 10-25 $\mu \mathrm{g}$ were administered during general anaesthesia. At the end of anaesthesia, patients received $1 \mathrm{~g}$ of intravenous paracetamol and, if necessary, $100 \mathrm{mg}$ of intravenous tramadol. During the wake-up period and hospitalisation, $1 \mathrm{~g}$ of intravenous then oral paracetamol $3 \times /$ day was administered. Then 
when necessary, following a regular visual analog scale (VAS) pain evaluation, $100 \mathrm{mg}$ of intravenous tramadol were administered $3 \times /$ day, then orally $50 \mathrm{mg} 3-4 \times$ /day over $2-5$ days, or nefopam $20 \mathrm{mg} 4 \times /$ day and when necessary, $5 \mathrm{mg}$ of subcutaneous morphine. At home, paracetamol per os and tramadol per os were prescribed, if necessary.

\section{Evaluation}

The evaluation was conducted prospectively in both groups, with same methodology and same analgesia protocol. The main factors investigated were the duration of the hospital stay, pain before and after surgery, symptomatic seroma and needle aspiration. Symptomatic seroma was defined as an axillary fluid collection inducing discomfort or pain with a medical consultation and eventually a needle aspiration. Only symptomatic seromas were recorded and patients requiring at least 1 aspiration were recorded as having had a needle aspiration. Pain was assessed using the classic 11-point VAS from $0=$ no pain to $10=$ the worst pain one could imagine [11]. Pain assessment was conducted pre-operatively and after surgery once daily until discharge from hospital, and finally at 6 weeks by a questionnaire sent by post. In the padding group alone, an evaluation of shoulder movement was performed by a physiotherapist at 6-8 weeks.

\section{Statistical Analysis}

Results for continuous variables were expressed as means (SD or range). A comparison of group characteristics was performed using $\chi^{2}$ or Fisher's exact tests. A p value $<0.05$ was considered statistically significant.

\section{Results}

Patient characteristics are summarised in table 1. There was no selection criterion except for conservative treatment for invasive breast cancer with an axillary lymphadenectomy through a separate incision from that of the tumourectomy. Indeed, no difference was observed between the 2 groups in clinical characteristics and breast cancer stage. The same mean number of removed nodes (14.4 vs. 13.5) indicates that axillary clearance was not less extensive in the padding group. The main factors investigated are summarised in table 2 and 3. The mean hospital stay was 2.4 (range, 1-4) days in the padding group and 4.2 (range 2-9) days in the drainage group $(\mathrm{p}<0.05)$. In the padding group there were fewer cases of seroma (11.4 vs. $23 \% ; p=0.01)$ and fewer needle aspirations for seroma ( 8.8 vs. $23 \%$; $=0.002$ ). The seroma rate was neither related to the body mass index (BMI) index nor to high blood pressure in either group. There was more suture line breakdown and less axillary infection in the padding group but the differences were not significant. The mean pain intensity at day 1 was $2.2(0-7)$ in the padding group and $2.8(0-8)$ in the drainage group $(\mathrm{p}=0.10)$ (table 3$)$. At 6 weeks, we obtained about two thirds of completed questionnaires in both groups. Only $28 \%$ of the patients reported some pain in the padding group versus $51 \%$ in the drainage group and the mean intensity was 3 and 4.3 , respectively $(\mathrm{p}<0.0001)$. Regarding the patients who came to hospital, the pain analysis indicated a large majority of neuropathic pain in both groups. Upper arm pain and mobility at 6 weeks in the padding group were acceptable: external rotation was in the range of normal mobility whereas abduction and antepulsion were limited in half of the patients.

Table 1. Distribution of the population

\begin{tabular}{llll}
\hline & Padding group $(\mathrm{n}=114)$ & Drainage group $(\mathrm{n}=185)$ & $\mathrm{p}$ \\
\hline Inclusion period & $01 / 01 / 2004$ to $03 / 30 / 2005$ & $01 / 01 / 2003 /$ to $12 / 31 / 2003$ & \\
Mean age (range), years & $56(24-84)$ & $58(27-85)$ & 0.76 \\
Obese* patients, \% & $13(11.4)$ & $19(10.3)$ & 0.76 \\
Mean pathological tumour size (SD), mm & $20.9(9.4)$ & $23.1(10.3)$ & 0.06 \\
Mean number of nodes removed (SD) & $14.4(4.9)$ & $13.5(4.7)$ & 0.12 \\
Involved nodes, $\mathrm{n}(\%)$ & $62(54.3)$ & $87(47)$ & 0.22 \\
Neo-adjuvant chemotherapy, $\mathrm{n}(\%)$ & $14(12.3)$ & $25(13.5)$ & 0.76 \\
\hline *Body mass index $>30 \mathrm{~kg} / \mathrm{m}^{2}$. & & \\
\hline
\end{tabular}

Table 2. Main results

\begin{tabular}{lccc}
\hline & Padding group $(\mathrm{n}=114)$ & Drainage group $(\mathrm{n}=185)$ & $\mathrm{p}$ \\
\hline Mean hospital stay, days & 2.4 & 4.2 & \\
Seroma, $\mathrm{n}(\%)$ & $13(11.4)$ & $43(23)$ & 0.01 \\
Needle aspiration, $\mathrm{n}(\%)$ & $10(8.8)$ & $43(23)$ & 0.002 \\
Suture breakdown, $\mathrm{n}(\%)$ & $3(2.6)$ & $3(1.6)$ & 0.54 \\
Axillary infection, $\mathrm{n}(\%)$ & $1(0.87)$ & $3(1.6)$ & 0.59 \\
\hline
\end{tabular}

Table 3. Pain evaluation

\begin{tabular}{lccc}
\hline & Padding group $(\mathrm{n}=114)$ & Drainage group $(\mathrm{n}=185)$ & $\mathrm{p}$ \\
\hline Mean VAS score* (range) & & & \\
Preoperative & $0.9(0-2)$ & $1.0(0-3)$ & 0.22 \\
Day 1 after surgery & $2.2(0-7)$ & $2.8(0-8)$ & 0.10 \\
6 weeks after surgery & $3.0(0-7)$ & $4.3(0-8)$ & $<0.0001$ \\
Patients with pain at 6 weeks, n (\%) & $32(28)$ & $94(51)$ & 0.001 \\
\hline
\end{tabular}

* Scale is from 0 to 10 . 


\section{Discussion}

The axillary padding technique makes it possible to avoid the post-operative space created by the lymphadenectomy. Furthermore, axillary flap adhesion is rapid which stops swelling and one can dispense with post-operative drainage. Muscular padding was first described by Aitken in 1984 [12] after mastectomy and lymphadenectomy and adapted for conservative treatment by Hamy in 1990 [13]. In 2004, we published a prospective evaluation of this muscular padding in a series of 152 patients. It was very effective but caused far more post-operative pain than the suction drain. Even if the late sequelae did not increase at 3.5 years, this intense additional pain led us to advise against the use of muscular padding [14]. Then the technique was modified using an aponeurosis or fascial padding which was first described by Classe and yielded good results [10]. Classe carried out a monocentric randomised comparison of fascial padding versus drainage in a series of 100 patients [15]. He demonstrated that axillary padding significantly reduced the mean length of hospital stay (1.8 vs. 4.5 days, $\mathrm{p}<0.001)$. Postoperative complications, pain, shoulder mobility and quality of life were similar in the 2 groups. Due to the small population size, it was not possible to assume equivalence of postoperative morbidity between padding and drainage in that study. A large difference would obviously have been demonstrated, had that been the case. Thus, those results show good feasibility and probably no wide differences in postoperative events between padding and drainage. The present study was conducted around the same period. This non-randomised comparison between padding and drainage in 299 patients demonstrates less pain and fewer post-operative complications in favour of the padding procedure. The hospital stay was shorter. Shoulder movements at 6 weeks were slightly affected in the padding group. Without a randomised comparison, we cannot prove that padding is associated with less pain than a suction drain. However, given the size of the population and the methodology in the same institution, we would have observed a substantial increase in pain or seroma formation if such had been the case. Our results are consistent with those in Classe's series. There is no other publication on aponeurosis padding after axillary surgery. Besides padding, the other options for solving the problem of prolonged drainage are disappointing or have not been well evaluated. External compression dressing fails to decrease the amount of drainage and may increase seroma formation [16]. Delayed shoulder exercise has no impact on the drainage volume and is associated with adverse post-operative shoulder dysfunction [17]. Multiple drainages, and a high or low vacuum intensity do not yield any compelling results $[9,18]$. Fibrin glue was evaluated in small series only, with conflicting results $[19,20]$. Earlier drain removal is feasible but is associated with varying rates of late seroma and needle aspirations in many series [1, 3, 5, 21]. Many institutions currently propose that their patients leave hospital rapidly with the drain still in place $[6,9]$.

\section{Conclusion}

After axillary lymphadenectomy, the management of postoperative seroma is at the crossroads of modern post-operative care. Early removal of the drain and, if unfeasible, early discharge with the drain in situ is the most frequent solution for many teams. However, this option is not always applicable and generates extra-cost for external care. Clearly, the best solution would be not to use a drain at all, if feasible. Among the potential alternative options, axillary aponeurosis padding seems be the best candidate for the moment. It is very simple and easy to achieve. This study confirmed the safety and feasibility of the technique. Large-scale multicentric evaluations are now warranted, in order to establish whether this technique could become a new standard to replace the suction drain.

\section{Acknowledgements}

The authors thank A. Rey, P. Martinelli and G. Baron-Merle for their help in conducting this study and L. Saint-Ange for editing.

\section{Disclosure Statement}

All authors certify they do not have any conflict of interest.

\section{References}

1 Kopelman D, Klemm O, Bahous H, Klein R, Krausz M, Hashmonai M: Postoperative suction drainage of the axilla: for how long? Prospective randomised trial. Eur J Surg 1999;165(2):117-120.

2 Zavotsky J, Jones RC, Brennan MB, Giuliano AE: Evaluation of axillary lymphadenectomy without axillary drainage for patients undergoing breast-conserving therapy. Ann Surg Oncol 1998;5(3):227-231.
Inwang R, Hamed H, Chaudary MA, Fentiman IS: A controlled trial of short-term versus standard axillary drainage after axillary clearance and iridium implant treatment of early breast cancer. Ann R Coll Surg Engl 1991;73(5):326-328.

4 Salmon RJ, Cody HS, Vedrenne JB, Asselain B, Durand JC, Pilleron JP: [Prevention of postoperative lymphocele after breast amputation]. Presse Med 1985;14(1):27-29.
5 Vinton AL, Traverso LW, Jolly PC: Wound complications after modified radical mastectomy compared with tylectomy with axillary lymph node dissection. Am J Surg 1991;161(5):584-588.

6 Bundred N, Maguire P, Reynolds J, Grimshaw J, Morris J, Thomson L et al.: Randomised controlled trial of effects of early discharge after surgery for breast cancer. BMJ 1998;317(7168):1275-1279. 
$>7$ Arnaud S, Houvenaeghel G, Julian-Reynier C, Protiere C, Moatti JP: [Women's preferences for early discharge after conservative breast surgery: feasibility, patient profile and satisfaction]. Ann Chir 2003;128(1):26-33.

8 Marchal F, Dravet F, Classe JM, Campion L, Francois T, Labbe D et al.: Post-operative care and patient satisfaction after ambulatory surgery for breast cancer patients. Eur J Surg Oncol 2005;31(5):495-499.

9 Pogson CJ, Adwani A, Ebbs SR: Seroma following breast cancer surgery. Eur J Surg Oncol 2003;29(9):711-717.

10 Classe JM, Dupre PF, Francois T, Robard S, Theard JL, Dravet F: Axillary padding as an alternative to closed suction drain for ambulatory axillary lymphadenectomy: a prospective cohort of 207 patients with early breast cancer. Arch Surg 2002;137(2):169-172.

11 Huskisson EC: Measurement of pain. Lancet 1974;2(7889):1127-1131.
12 Aitken DR, Hunsaker R, James AG: Prevention of seromas following mastectomy and axillary dissection. Surg Gynecol Obstet 1984;158(4):327-330.

13 Garnier JM, Hamy A, Classe JM, Laborde O, Sagot P, Lopes P et al.: A new approach to the axilla: functional axillary lymphadenectomy and padding. J Gynecol Obstet Biol Reprod (Paris) 1993;22(3):237-242.

14 Garbay JR, Picone O, Baron-Merle G, Yacoub S, Lasry S, Missana MC et al.: Axillary lymphadenectomy with muscular padding, without drainage. Gynecol Obstet Fertil 2004;32(12):1039-1046.

15 Classe JM, Berchery D, Campion L, Pioud R, Dravet F, Robard S: Randomized clinical trial comparing axillary padding with closed suction drainage for the axillary wound after lymphadenectomy for breast cancer. Br J Surg 2006;93(7):820824.

16 O'Hea BJ, Ho MN, Petrek JA: External compression dressing versus standard dressing after axillary lymphadenectomy. Am J Surg 1999;177(6):450453.
17 Christodoulakis M, Sanidas E, de BE, Michalakis J, Volakakis E, Tsiftsis D: Axillary lymphadenectomy for breast cancer - the influence of shoulder mobilisation on lymphatic drainage. Eur J Surg Oncol 2003;29(4):303-305.

8 Bonnema J, van Geel AN, Ligtenstein DA, Schmitz PI, Wiggers T: A prospective randomized trial of high versus low vacuum drainage after axillary dissection for breast cancer. Am J Surg 1997;173(2):76-79.

19 Langer S, Guenther JM, DiFronzo LA: Does fibrin sealant reduce drain output and allow earlier removal of drainage catheters in women undergoing operation for breast cancer? Am Surg 2003;69(1):77-81.

20 Jain PK, Sowdi R, Anderson AD, MacFie J: Randomized clinical trial investigating the use of drains and fibrin sealant following surgery for breast cancer. Br J Surg 2004;91(1):54-60.

21 Liu CD, McFadden DW: Overnight closed suction drainage after axillary lymphadenectomy for breast cancer. Am Surg 1997;63(10):868-870. 